\title{
Abstract Index
}

\author{
8IKC Extended Abstracts - Table of Contents - alphabetical by Author(s)
}

RETURN TO

HOME PAGE

\author{
Red Abstract numbers are linked to Abstract
}

Authors

Abstract Title

File Name (.pdf)

M Anand, LA Taylor, WD Carlson, D-H Taylor, NV Sobolev

Diamond Genesis Revealed by X-Ray Tomography of

Diamondiferous Eclogites

Keywords: HRCXT, Udachnaya, Yakutia, macro-diamond, kimberlite, metasomatism

FLA_0083

M Anand, LA Taylor, KC Misra, WD Carlson, NV Sobolev Diamondiferous Eclogite Dissections: Anomalous Diamond Genesis?

Keywords: diamond inclusions, Udachnaya, Yakutia,

macrodiamond, kimberlite, metasomatism, in situ analysis,

cathodoluminescence

FLA 0084

EC Anckar, JJ Gurney, C Thiart

A Statistical Approach to Finger-Print Run-of-Mine

Diamonds using FTIR Spectra, Size Distributions and

Physical Characteristics

Keywords: nitrogen, cathodoluminescence, database, diamond populations, discriminant analysis

FLA_0092

CM Appleyard, AP le Roex, DR Bell

The Geochemistry of a Suite of Eclogite Xenoliths from the

Rietfontein Kimberlite, South Africa

Keywords: off-craton, petrography, mantle derived, basaltic,

oxygen isotopes, subduction

FLA_0044

CM Appleyard, KS Viljoen, R Dobbe

A Study of Eclogitic Diamonds and their Inclusions from the Finsch Kimberlite Pipe, South Africa

Keywords: syngenetic, Type IaAB, geothermometry,

cathodoluminescence, octahedral growth

FLA_0045

DP Araujo, JC Gaspar, Y Fei, EH Hauri, R Hemley, GP Bulanova Mineralogy of Diamonds from the Juina Province, Brazil

Keywords: diamond, Juina, lower mantle, transition zone, morphology, cathodoluminescence, Ir, SIMS

FLA_0279

KA Armstrong, T Nowicki, GH Read

Kimberlite AT-56: A Mantle Sample from the North Central Superior Craton, Canada

Keywords: Attawapiskat, websterite, eclogite, Ni thermometry FLA_0277
Authors

Abstract Title

File Name (.pdf)

JP Armstrong

Diamond Discovery in the Slave Craton: Compilations of Exploration Data as Tools for Future Discovery

Keywords: data base, indicator mineral, drill log, magnetic, compilation

FLA_0190

JP Armstrong, RL Barnett

The Association of $Z N$-Chromite with Diamondiferous

Lamprophyres and Diamonds: Unique Compositions as a

Guide to the Diamond Potential of Non-Traditional

Diamond Host Rocks

Keywords: chromite-in-diamond, Wawa, Yellowknife, spinel, amphibole

FLA_0230

JP Armstrong, M Wilson, RL Barnett, T Nowicki, BA Kjarsgaard Paragenesis of Primary Magmatic Sr-Ba-Mg-Ca Carbonates from Ultra-Fresh Hypabyssal Kimberlite, Lac de Gras Kimberlite Field, Slave Craton, NWT Canada

Keywords: monticellite, spinel, calcite, segregation, solvus,

dolomite

FLA_0189

F Arnott, EO Kostlin

Petrophysics of Kimberlites

Keywords: geophysical exploration, physical properties, magnetics, density, resistivity, seismic velocity, gamma radiation

FLA_0389

IV Ashchepkov, NV Vladykin, NP Pokhilenko, NV Sobolev, EV Malygina, SG Kuligin, YI Ovchinnikiv, NP Afanasiev, GM Mkrtychan, AY Rotman, LM Pokhilenko, AM Logvinova, OS Khmel'nikova, SI Kostrovitsky, AV Tolstov

Clinopyroxene Geotherms for the Mantle Columns Beneath Kimberlite Pipes from Siberian Craton

Keywords: geotherm, thermobarometry, layering, garnet, pyrope, ilmenite, geochemistry, Siberian craton FLA_0355

S Aulbach, WL Griffin, SY O'Reilly, TE McCandless

The Lithospheric Mantle Beneath the Buffalo Head Terrane, Alberta : Xenoliths from the Buffalo Hills Kimberlites

Keywords: lithospheric mantle, peridotite, xenolith, xenocryst, metasomatism, Archean, Buffalo Head Terrane, Alberta FLA_0010 
Authors

Abstract Title

File Name (.pdf)

S Aulbach, WL Griffin, NJ Pearson, SY O'Reilly, K Kivi, BJ Doyle Origins of Eclogites Beneath the Central Slave Craton Keywords: xenoliths, mantle, subduction, rutile, Hafnium, Carbon, Lac de Gras

FLA_0011

JA Ayer, DA Wyman

Origin of Diamondiferous Archean Lamprophyres in the Evolution of the Michipicoten and Abitibi Greenstone Belts Keywords: Superior Province, Wawa area, Cobalt area, petrogenesis, subduction, stability field, metastable FLA_0291

WP Barnett

Subsidence Breccias in Kimberlite Pipes: An Application of a Fractal Analysis

Keywords: volcanology, Venetia, size distribution, shape analysis, statistics

FLA_0314

IB Basson, G Viola

Passive Kimberlitic Fluid Emplacement into Dilating Dyke-Fracture Systems in South Africa

Keywords: kimberlite, South Africa, Group II, dykes, structural, tectonic, emplacement mechanisms, dikes FLA 0387

M Baumgartner, E Ankar, H Grutter

Compositional Classification of 'Kimberlitic' and 'NonKimberlitic' Ilmenite, with Implications for Visual Selection and Discrimination

Keywords: kimberlite, lamprophyre, exploration, oxidation FLA_0281

DR Bell, M Gregoire, TL Grove, ND Chatterjee, SA Bowring Silica and Carbon Deposition in Kimberley Peridotites Keywords: metasomatism, diamond, magnesite, orthopyroxene, fluid, Archean, mantle, tectosphere FLA 0289

P Berendsen, RL Cullers, A Barczuk, A Al-Ammar New Data on Kimberlites and Lamproites in Eastern Kansas, U.S.A.

Keywords: structure, drill hole information, morphology, petrography

FLA 0288

AK Berryman, BH Scott Smith, BC Jellicoe

Geology and Diamond Distribution of the 140/141

Kimberlite, Fort a la Corne, Central Saskatchewan, Canada Keywords: grade, pyroclastic, deposit, evaluation, drilling, drill vent

FLA_0373
Authors

Abstract Title

File Name (.pdf)

TC Birkett, TE McCandless, CT Hood

Petrology of the Renard Igneous Bodies: Host Rocks for Diamond in the Northern Otish Mountains Region, Quebec Keywords: kimberlite, diatreme, exploration, mineralogy, melnoite

FLA_0215

AV Bobrov, Yu. Litvin, V Butvina

Diamond Synthesis in Sulfide Medium at 6-8 Gpa: Application to Natural Data

Keywords: experiment, pressure, nucleation, crystal, sulphide FLA_0145

AV Bobrov, EM Verichev, VK Garanin, KV Garanin,

GP Kudryavtseva

Xenoliths of Mantle and Metamorphic Rocks from the

Diamondiferous V.Grib Pipe: Petrology and Genetic Aspects Keywords: peridotite, pyroxenite, eclogite, geothermometer, geobarometer

FLA_0146

FR Boyd, KO Hoal, BG Hoal, DG Pearson, PH Nixon, MJ Kingston

Garnet Lherzolites from Louwrencia, Nambia: Bulk Compositions and P/T Relations

Keywords: xenolith, kimberlite, Kaapvaal, lithosphere, craton, osmium, thermobarometry, geotherm

FLA_0283

LP Boyer, CT Hood, TE McCandless, DN Skelton, RD Tosdal Volcanology of the Buffalo Hills Kimberlites, Alberta, Canada: Some Preliminary Observations

Keywords: crater, hypabyssal, facies, volcaniclastic, bedding, flow slignment, Cretaceous, magmaclast FLA_0253

FE Brenker, T Stachel, JW Harris

TEM-Analysis of Inclusions in Diamonds from the Lower Mantle and Transition Zone (Kankan District, Guinea) Keywords: olivine, fluid, dislocation, exsolution, deformation, clinopyroxene, garnet

FLA 0297

GP Brey, V Bulatov, A Girnis, J Harris, T Stachel

Ferropericlase - A Lower Mantle Phase in the Upper Mantle Keywords: olivine, perovskite, Na, carbonatite, reduction, Kankan FLA_0208

JA Brod, JC Gaspar, HS Diniz-Pinto, TC Junqueira-Brod Spinel Chemistry as an Indicator of Crystal Franctionation and Liquid Immiscibility in the Tapira Alkaline-Carbonatite Complex, Minas Gerais, Brazil

Keywords: carbonatite, chromite, magnetite, bebedourite, kamafugite, APIP, phoscorite, nelsonite

FLA_0335 
Authors

Abstract Title

File Name (.pdf)

JA Brod, TC Junqueira-Brod, JC Gaspar, SA Gibson,

RN Thompson

Ti-Rich and Ti-Poor Garnet from the Tapira Carbonatite

Complex, SE Brazil: Fingerprinting Fractional

Crystallisation and Liquid Immiscibility

Keywords: melanite, schorlomite, andradite, liquid immiscibility,

APIP, bebedourite, kamafugite, phlogopite picrite, crystallization

FLA_0339

S Buhre, HK Steinberg, GP Brey, SM Clark

Trace Element Solubility and Reaction Kinetics in the CAS-System

Keywords: Ca-perovskite, Ca-walstromite, Ca-silicates,

diamonds, REE, in situ

FLA_0163

GP Bulanova, E Muchemwa, DG Pearson, BJ Griffin, S Kelly,

S Klemme, CB Smith

Syngenetic Inclusions of Yimengite in Diamond from Sese

Kimberlite (Zimbabwe) - Evidence for Metasomatic

Conditions of Growth

Keywords: alkali-titanate, chromite, Ar/Ar-dating,

metasomatism, upper mantle

FLA 0123

GP Bulanova, DG Pearson, EH Hauri, HJ Milledge,

YuP Barashkov

Dynamics of Diamond Growth: Evidence from Isotope and FTIR Trends

Keywords: carbon, nitrogen, eclogite, peridotite, mantle

FLA_0125

R Burgess, D Harrison, E Hobson, JW Harris

Noble Gas and Halogen Constraints on the Origin of

Volatile-Rich Fluids in Canadian Diamonds

Keywords: diamond, mantle fluid, halogen, noble gases, Canada FLA 0155

R Burgess, G Kiviets, JW Harris

Different Age Populations of Eclogitic Diamonds in the

Venetia Kimberlite: Evidence from Ar-Ar Dating of

Syngenetic Clinopyroxene Inclusions

Keywords: diamond, argon, age, dating, Africa

FLA_0162

D Bush, F Nel, C Revering, M Kirkley

Geostatistical Methods Employed in Resource Evaluation of

the Snap Lake Diamond Deposit, NWT, Canada

Keywords: estimation, kriging, simulation

FLA_0264
Authors

Abstract Title

File Name (.pdf)

D Canil

Peridotites, Garnets and Trace Elements: A Telling Trilogy about Mantle Lithosphere

Keywords: peridotite, garnet, trace elements, lithosphere, kimberlite

FLA_0131

MP Carlson, SB Treves

Characterization and Geologic Setting of the Elk Creek

Carbonatite, Southeast Nebraska, USA

Keywords: geophysics, gravity, magnetics, Kansas

FLA_0009

RW Carlson, AJ Irving, DJ Schulze, BC Hearn Jr.

Timing of Lithospheric Mantle Modification Beneath the

Wyoming Craton

Keywords: Wyoming Craton, peridotite xenoliths, isotopic

composition, elemental composition, age

FLA_0185

P Cartigny, T Stachel, J W Harris, M Javoy

$\mathrm{C}$ - and $\mathbf{N}$-Stable Isotope Characterics of Diamonds from

Namibia

Keywords: diamond, stable isotopes, carbon, nitrogen,

Namibia, alluvials

FLA_0122

DMDeR Channer, A Egorov, FV Kaminsky

Geological and Tectonic Setting of the Guaniamo Kimberlite

Sheets, South - West Venezuela

Keywords: diamond, eclogitic, proterozoic, subduction

FLA_0157

JG Chapman, GL Boxer

Size Distribution Analyses for Estimating Diamond Grade and Value

Keywords: diamonds, micro-diamonds, grade, size

distributions, frequencies, value

FLA 0274

IL Chinn, C Pienaar, CL Kelly

Diamond Growth Histories at Premier Mine

Keywords: cathodoluminescence, infra-red spectroscopy, Type

II diamonds, zonation

FLA_0159

NIJ Coe, AP le Roex, JJ Gurney

The Petrology and Geochemistry of the Swartruggens and Star Kimberlite Dyke Swarms, South Africa

Keywords: Group II kimberlite, dike, petrography, trace elements, major elements, dyke

FLA 0118 
Authors

Abstract Title

File Name (.pdf)

HO Cookenboo

Extension of Ultradepleted Mantle within the Contwoyto

Terrane of the Slave Craton, Northern Canada

Keywords: Snap Lake, Gahcho Kue, Diavik, Ekati, garnet, G10, chemistry

FLA_0202

SA Cooper, CM MacRae, NC Wilson, NVY Scarlett, WT Marx

Diamond Coatings that Affect Diamond Recoveries on Grease Tables Investigated by an Electron Microprobe Keywords: cathodoluminescence, Perdevlei, Kareevlei Wes, orangeite, geochemistry, Barkley West

FLA 0210

B Corner

Geophysical Mapping of Major Structures of Southern Africa and an Assessment of Kimberlite Correlation

Keywords: geophysics, regional, structure, kimberlites, correlation, southern Africa

FLA_0245

VS Costa, JC Gaspar, MM Pimentel

Peridotite and Eclogite Xenoliths from the Juina Kimberlite Province, Brazil

Keywords: diamond, Sao Luiz, Amazon Craton, Sm-Nd, mineral chemistry, geothermometry

FLA_0336

N Coussaert, M Gregoire, JCC Mercier, DR Bell, D Demaiffe, AP Le Roex, L André

The Origin of Clinopyroxene in Cratonic Mantle

Keywords: metasomatism, Kaapvaal, MARID, PIC, garnet lherzolite

FLA_0383

N Coussaert, J-C-C Mercier, D Demaiffe, L Andre

Equilibrium conditions revisited for Lesotho kimberlites

Keywords: thermodynamic, thermobarometry, metasomatism, solvus orthopyroxene, inversion, lherzolite, harzburgite, CMS FLA_0266

DC Crabtree, TF Morris, JA Ayer, RP Sage

The Identification of Lherzolitic Cr-Diopsides in Kimberlites Exploration Programs: Examples from Indicator Mineral Surveys carried out in the Province of Ontario Keywords: jadeite, kosmochlor, lamprophyre, Kapuskasing Structural Zone

FLA 0302

J Crawford, HH Helmstaedt

Comparative Study of Hypabyssal Kimberlite from Four Locations within the Slave Craton

Keywords: Finlay, Muskox, Rich, Jean, petrography, mineral chemistry, geochemistry

FLA_0128
Authors

Abstract Title

File Name (.pdf)

RA Creaser, H Grutter, J Carlson, B Crawford

Macrocrystal Phlogopite Rb-Sr Dates for the Ekati Property

Kimberlites, Slave Province, Canada: Evidence for Multiple

Intrusive Episodes in the Paleocene and Eocene

Keywords: geochronology, age dating, emplacement age, isotope FLA_0204

SD Creighton, DR Eccles

A Preliminary Study of the Mineral Chemistry of Selected Alberta Kimberlites

Keywords: phlogopite, spinel, olivine, Buffalo Head Hills,

Birch Mountains

FLA_0012

C Dalpe, DJ Ballantyne

Diamond Profiling: A New Approach for Forensic Application Keywords: gem, data base, fingerprinting, LA-ICP-MS, RCMP FLA_0348

LRM Daniels

The Genesis of Kimberlite Concentrate Xenocrystic Spinels

Keywords: Kaapvaal, magmatic, residue, subsolidus, TiO2-rich, TiO2-poor

FLA 0259

GR Davies, AJ Stolz, IL Mahotkin, GM Nowell, DG Pearson Trace Element and Sr-Pb-Nd-Hf Isotope Evidence for Ancient Fluid-Related Enrichment in the Source Region of Aldan Shield Lamproites

Keywords: HFSE depletion, HFSE fractionation, Nd-Sr-Pb-Hf isotopes, metasomatism

FLA_0256

RM Davies, WL Griffin, SY O'Reilly, BJ Doyle

Geochemical Characteristics of Microdiamonds from

Kimberlites at Lac de Gras, Central Slave Craton

Keywords: diamond, eclogitic, peridotitic, ultradeep, mineral inclusions, carbon isotopes, nitrogen aggregation states, nitrogen contents

FLA_0048

RM Davies, WL Griffin, SY O'Reilly, TE McCandless Inclusions in Diamonds from the K10 and K14 Kimberlites, Buffalo Hills, Alberta, Canada: Diamond Growth in a Plume? Keywords: diamond inclusions, eclogitic, peridotitic, ultradeep, carbon isotopes, nitrogen contents, nitrogen aggregation states

FLA_0049

JB Dawson

A Harzburgite to Garnet Lherzolite Transformation:

The Possible Roles of Strain and Metasomatism

Keywords: olivine, enstatite, garnet, spinel, geochemistry, trace elements, peridotite

FLA_0013 
Authors

Abstract Title

File Name (.pdf)

I Dencker, P Nimis, A Zanetti, NV Sobolev

Major and Trace Elements Composition of Cr-Diopsides

from the Zagadochnaya Kimberlite Pipe (Yakutia, Russia):

Insights into Metasomatic Processes in the Yakutian

Lithosphere

Keywords: diamond, Siberia, mantle, metasomatism

FLA 0234

DP Dowall, DG Pearson, GM Nowell, BA Kjarsgaard,

J Armstrong, MSA Horstwood

Comparative Geochemistry of Kimberlites from the Lac de Gras Field, NWT - An Integrated Isotopic and Elemental Study

Keywords: Slave province, Hf isotopes, $\mathrm{Nd}$ isotopes, mantle array, transitional

FLA_0153

PM Doyle, JJ Gurney, A le Roex

Xenoliths from the Arnie, Misery and Pigeon Kimberlites, Ekati Mine, NWT, Canada

Keywords: major elements, trace elements, Ekati, mantle, geotherm, modeling, kimberlite, Slave

FLA 0090

D Dunn, D Smith, SC Bergman

Mantle Xenoliths from the Prairie Creek Lamproite

Province, Arkansas, USA

Keywords: geotherm, depletion, lherzolite, tectonics, diamond FLA_0119

D Dunn

Diamond Evaluation of the Prairie Creek Lamproite

Province, Arkansas, USA

Keywords: grade, erosion, stratigraphy, eluvial, alluvial, mining FLA 0380

DR Dyck, PA Oshust, JA Carlson, TE Nowicki, MP Mullins

Effective Resource Estimates for Primary Diamond

Deposits from the Ekati Diamond Mine, Canada

Keywords: Lac de Gras, economic, kimberlite, volumetrics, density, geostatistics

FLA 0196

DR Eccles, LM Heaman, RW Luth, RA Creaser

Petrogenetic Considerations for the Late Cretaceous

Northern Alberta Kimberlite Province

Keywords: volcaniclastic kimberlite, Mountain Lake, Buffalo

Head Hills, Birch Mountains, petrogenesis

FLA_0041

J Ekkerd, J Stiefenhofer, M Field, P Lawless

The Geology of Finsch Mine, Northern Cape Province,

South Africa

Keywords: Kaapvaal, Karoo, dike, mine plan, geology, dyke

FLA_0310
Authors

Abstract Title

File Name (.pdf)

EI Erlich, WD Hausel

Timing of Kimberlite Magmatism and Different Types of Diamond-bearing Complexes

Keywords: timing, kimberlites, lamproites, tectonics, astroblemes

FLA_0361

Y Fedortchouk, D Canil, JA Carlson

Oxygen Fugacity of Kimberlite Magmas and their

Relationship to the Characteristics of Diamond

Populations, Lac de Gras, N.W.T., Canada

Keywords: crystallization temperature, intensive variables, diamond morphology, resorption, etching, dissolution, diamond quality, redox regime, crystallisation FLA_0098

MM Fenton, JG Pawlowicz, RC Paulen, GJ Prior, RA Olsen Quaternary Geology of Northern Alberta: Implications for Kimberlite Exploration

Keywords: drift prospecting, Buffalo Head Hills, Birch Mountains, ice flow mapping, Mountain Lake, glacial sediments, bedrock topography, drift thickness

FLA_0365

SF Foley, AV Andronikov

The Genesis of Ultramafic Lamprophyres

Keywords: rifts, experiments, kimberlite, aillikite, alnoite, carbonatite

FLA_0236

SF Foley, S Buhre, DE Jacob, T Rehfeldt

Pyroxenite and Dunite Xenoliths as Metamorphosed

Cumulates from the Archean Lower Ocean Crust

Keywords: subduction, eclogite, cratonic lithosphere, crustal formation

FLA_0235

D Francis

Implications of Major Element Composition for the Mantle Sources of Kimberlite, Aillikite, Olivine Lamproiite, and Meimechite

Keywords: diamond, carbonate, Fe, picrite, phlogopite FLA_0248

J Ganga, A Rotman, S Nosiko

Pipe Catoca, an Example of the Weakly Eroded Kimberlites from North-East of Angola

Keywords: crater, diatreme, diamond, pyrope

FLA 0312 
Authors

Abstract Title

File Name (.pdf)

BP Garden, RW Carlson, SB Shirey, JJ Gurney

RE-OS Systematics of Lithospheric Peridotites \& Eclogites

from the Bobbejaan \& Bellsbank Dykes, Kaapvaal Craton, South Africa

Keywords: isotopes, xenoliths, Kimberley, Group II kimberlite, dikes

FLA 0147

JC Gaspar, ALN Araujo, RW Carlson, SE Sichel, JA Brod, PB Sgarbi, JCM Danni

Mantle Xenoliths and New Constraints on the Origin of Alkaline Ultrapotassic Rocks from the Alto Paranaiba and Goias Igneous Provinces, Brazil

Keywords: mantle xenoliths, isotopic data, Alto Paranaiba Igneous Province, Goiás Alkaline Province, kamafugites, kimberlites, Brazil

FLA 0337

A Girnis, HS Grutter

Thermobarometry of Mantle Peridotites: Calibration Based on Experimental and Natural Data

Keywords: pressure, temperature, thermodynamic models, 5 -phase peridotite, xenolith

FLA 0250

J Glinnemann, K Kusaka, J Harris, B Bleisteiner, B Winkler Oriented Graphite Single-Crystal Inclusions in Diamond Keywords: optical microscopy, X-ray diffraction, Micro-Raman spectroscopy, mutual orientation, coincidence site lattice, remnant pressures, crystallography

FLA_0354

AV Golovin, VV Sharygin, NP Pokhilenko, VG Mal'kovets, NV Sobolev

Secondary Melt Inclusions in Olivine from Unaltered Kimberlites of the Udachnaya-Eastern Pipe, Yakutia

Keywords: Raman, EPMA, alkaline, carbonatite, phlogopite, chlorides, sulphates, Palabora

FLA_0226

AM Gonzales, M Baumgartner, K Gelo

The Ranch Lake Indicator Mineral Train: Single or Multiple Sources?

Keywords: Slave, dispersion, kimberlite, till, abundance,

kelyphite

FLA_0198

S Graham, DD Lambert, SR Shee

Geochemical and Isotopic Evidence of a Kimberlite-MelnoiteCarbonatite Genetic Link

Keywords: melnoite, kimberlite, carbonatite, Re-Os, Sm-Nd, Yilgarn, Australia, isotopes

FLA_0130
Authors

Abstract Title

File Name (.pdf)

WL Griffin, SY O'Reilly, BJ Doyle, Kevin Kivi, HG Coopersmith Lithospheric Mapping Beneath the North American Plate Keywords: lithosphere structure, North America cratons, Archean mantle roots, lithosphere evolution, Michigan mantle section, Slave craton, North America mantle domains, mantle seismic velocity

FLA_0070

HS Grutter, AH Menzies

Mutually Consistent Classification Schemes for Mantle-

Derived Garnet and Chromite, for use by Diamond

Explorers

Keywords: mineralogy, petrology, peridotite, eclogite,

websterite, graphite, G10, G9

FLA_0385

HS Grutter, RO Moore

Pyroxene Geotherms Revisited - An Empirical Approach Based on Canadian Xenoliths

Keywords: thermobarometry, garnet peridotite, Somerset Island, Kirkland Lake, Slave craton, North Lesotho, Gibeon,

Kimberley

FLA_0272

GH Gudfinnsson, DC Presnall

Continuous Gradations among Primary Kimberlitic, Carbonatitic, Melilititic, and Komatiitic Melts in Equilibrium with Garnet Lherzolite at 3-8 Gpa Keywords: kimberlite, carbonatite, melilitite, melting experiments, melt generation, $\mathrm{CO} 2$, multi-anvil FLA_0249

JJ Gurney, P Hildebrand, J Carlson, D Dyck, Y Fedortchouk Diamonds from the Ekati Core and Buffer Zone Properties Keywords: components, characteristics, morphology, colour, growth, resorption, color

FLA_0035

JJ Gurney, KJ Westerlund, RW Carlson, SB Shirey

Mineral Compositions and RE-OS Isotope Systematics of Harzburgitic Nodules from the Panda Kimberlite, Slave Craton

Keywords: lithosphere, melt-depletion, CSBC, Archean FLA_0138

JL Gurney, M Baumgartner, E Anckar, JJ Gurney, TE Nowicki, HS Grutter, M Coetzee, N Mason-Jones

Kimberlite Almanac

Keywords: mineral chemistry, petrography, diamonds, Finsch FLA_0316 
Authors

Abstract Title

File Name (.pdf)

SE Haggerty

India: Geological Setting and Chemistry of Kimberlite Clan Rocks in the Dharwar Craton

Keywords: India, Dharwar Craton, kimberlite clan rocks, Proterozoic, Rodinia, geochemistry, famous diamonds FLA_0029

AP Hamblin, LD Stasiuk, AR Sweet, GD Lockhart, DR Dyck, K Jagger, LR Snowdon

Post-Kimberlite Eocene Strata Within a Crater Basin, Lac de Gras, Northwest Territories, Canada

Keywords: sedimentology, organic petrology, palynology, rock eval, thermal maturity, peat, oil shale, vitrinite reflectance

FLA 0057

MA Hamilton, NV Sobolev, RA Stern, DG Pearson

SHRIMP U-Pb Dating of a Perovskite Inclusion in Diamond: Evidence for a Syneruption Age for Diamond Formation, Sytykanskaya Kimberlite Pipe, Yakutia Region, Siberia Keywords: geochronology, Alakit, ion microprobe, diamond growth, Schryburt Lake, syngenetic, peridotitic, $206 \mathrm{~Pb} / 238 \mathrm{U}$ FLA 0388

PTC Hammer, K Ramachandran, RM Clowes

Seismic Reflection Imaging of Thin, Kimberlite Dykes and Sills: Exploration and Deposit Characterization of the Snap Lake Dyke, Canada

Keywords: seismic, reflection, resolution, detection, feathering, drill, dike

FLA_0058

M Hanrahan, T Stachel, GP Brey, Y Lahaye

Garnet Peridotite Xenoliths from the Koffiefontein Mine, South Africa

Keywords: lherzolite, garnet, clinopyroxene, geotherm, sheared peridotites, REE

FLA 0369

GE Harlow, R Davies

Status Report on Stability of K-Rich Phases at UpperMantle Conditions

Keywords: experimental, clinopyroxene, phase-X, K-richterite, K-cymrite, phlogopite, K-hollandite, phengite

FLA_0295

SE Harvey, BA Kjarsgaard, LI Kelley

Kimberlites of Central Saskatchewan: Complication and

Significance of Indicator Mineral Geochemistry with Respect to Diamond Potential

Keywords: indicator, indicator minerals, Saskatchewan, Fort a la Corne, geochemistry

FLA_0193
Authors

Abstract Title

File Name (.pdf)

PC Hayman, MG Kopylova, FV Kaminsky

Alluvial Diamonds from the Rio Soriso (Juina, Brazil)

Keywords: diamond, morphology, nitrogen, cathodo-

luminescence, fluorescence, Juina, Brazil, nitrogen aggregation FLA_0320

JW Head, L Wilson

Diatremes and Kimberlites 1: Definition, Geological Characteristics and Associations

Keywords: dikes, sill, root zone, carbon dioxide, xenoliths, peridotite, dykes

FLA_0287

LM Heaman, RA Creaser, HO Cookenboo, T Chacko

Multi-Stage Modification of the Mantle Lithosphere Beneath the Slave Craton: Evidence from an Unusual Suite of Zircon-Bearing Eclogite Xenoliths entrained in the Jericho Kimberlite, Canada

Keywords: Jericho kimberlite, eclogite xenolith, geochronology, Slave lithosphere, metasomatism FLA_0100

LM Heaman, BA Kjarsgaard

The Temporal Evolution of North American Kimberlite Keywords: geochronology, kimberlite genesis, emplacement patterns, tectonic processes

FLA_0099

BC Hearn Jr.

Upper-Mantle Xenoliths in the Homestead Kimberlite, Central Montana, USA: Depleted and Re-Enriched Wyoming Craton Samples

Keywords: garnet, spinel, phlogopite, clinopyroxene, ilmenite, thermobarometry, olivine, xenocryst

FLA_0126

AJ Hell, WRH Ramsay, G Rheinberger, S Pooley

The Geology, Age, Near Surface Features and Mineralogy of the Merlin Kimberlites, Northern Territory, Australia

Keywords: diatreme facies, textural phases, emplacement, infill sediments, subsidence, diamond potential

FLA_0273

A Henning, G Kiviets, S Kurszlaukis, E Barton, F Mayaga-Mikolo Early Proterozoic Metamorphosed Kimberlites from Gabon Keywords: Mitzic, meta-kimberlite, Congo Craton, Ayolo, Amie, Archean, Ar/Ar, U-Pb dating

FLA_0066

CM Hetman, BH Scott Smith, JL Paul, FW Winter

Geology of the Gahcho Kue Kimberlite Pipes, NWT, Canada: Root to Diatreme Transition Zones

Keywords: 5034, Hearne, Tuzo, Tesla, tuffisitic, hypabyssal, magmaclastic, fluidisation, fluidization, textures, emplacement FLA_0352 
Authors

Abstract Title

File Name (.pdf)

SL Hodder

Petrography and Mineral Chemistry of the McLean

Kimberlite, Lake Timiskaming, Ontario

Keywords: Canada, aphanitic, mineralogy

FLA 0170

HE Hoefer, GP Brey, AB Woodland

Iron Oxidation State of Mantle Minerals Determined from

L Emission Spectra by the Electron Microprobe

Keywords: speciation, EPMA, garnet, pyroxene, ferric iron,

$\mathrm{X}$-ray spectroscopy, valency, $\mathrm{Fe} 3+$

FLA 0173

CT Hood, TE McCandless

Systematic Variations in Xenocryst Mineral Composition at the Province Scale, Buffalo Hills Kimberlites, Alberta, Canada

Keywords: pyrope, chromite, ilmenite, proterozoic,

thermobarometry, indicators, diamond, lherzolite

FLA_0199

MT Hutchison, PH Nixon, SL Harley

Corundum Inclusions in Diamonds - Discriminatory

Criteria and a Corundum Compositional Database

Keywords: Sao Luiz, Juina, Brazil, ruby, sapphire, nickel, prospectivity, granulite

FLA_0069

AJ Irving, SM Kuehner, PC Ellsworth

Petrology and Thermobarometry of Mantle Xenoliths from the Eocene Homestead Kimberlite Pipe, Central Montana, USA

Keywords: garnet, peridotite, dunite, Grassrange, Wyoming craton, lithosphere

FLA_0050

TJ Ivanic, B Harte, SR Burgess, JJ Gurney

Factors in the Formation of Sinuous and Humped REE Patterns in Garnets from Mantle Harzburgite Assemblages

Keywords: Jagersfontein, fractional crystallisation,

disequilibrium, megacryst melt, percolation, diffusion, rare earth elements, equilibrium, crystallisation

FLA 0078

ES Izraeli, JW Harris, O Navon

Mineral Micro-Inclusions in Cloudy Diamonds from

Koffiefontein, South Africa

Keywords: carbonatitic, melt, fluid, peridotitic, eclogitic, mica FLA 0113

DE Jacob

Origin and Genesis of Eclogites from the Earth's Mantle

Keywords: review, ocean crust, cumulates, geochemistry, trace elements, isotopes

FLA_0238
Authors

Abstract Title

File Name (.pdf)

DE Jacob, A Fung, E Jagoutz, DG Pearson

Petrology and Geochemistry of Eclogite Xenoliths from the

Ekati Kimberlites Area

Keywords: eclogites, trace elements, Canada, isotopes

FLA_0239

E Jagoutz, R. Jotter

On the Search for Neodymium-142 in Terrestrial Rocks

Keywords: 142 Neodymium, primary differentiation, primitive earth, primitive lithosphere, extinct isotopes, SNC meteorites, cratonic lithosphere, cratonic mantle

FLA_0081

$\mathrm{J}$ Jakubec

Role of Geology in Project development

Keywords: diamond mine, geotechnics, kimberlite, contact zones FLA_0362

J Jakubec, L Long, T Nowicki, D Dyck

Underground Geotechnical and Geological Investigation at

the Ekati Mine - Koala North Case Study

Keywords: diamond mine, geotechnics, kimberlite, mudstones FLA_0363

PE Janney, AP le Roex, RW Carlson, DR Bell

Os and Hf Isotopic Constraints on the Origins of Olivine Melilitites from Western South Africa

Keywords: melilitite, kimberlite, Osmium, Hafnium, South

Africa

FLA_0127

AL Jaques, PR Milligan

Patterns and Controls on the Distribution of Diamond

Pipes in Australia

Keywords: exploration, geophysics, kimberlite, lamproite,

potential field, gravity, lithosphere, structure

FLA_0132

SM Jensen, K Secher, TM Rasmussen, T Tukiainen, JD Krebs,

F Schjøth

Distribution and Magnetic Signatures of Kimberlitic Rocks in the Sarfartoq Region, Southern West Greenland

Keywords: dykes, dikes, occurrences, geophysics, hyperspectral FLA_0140

LH Johnson, D Phillips

40Ar/39Ar Dating of Mantle Metasomatism: A Noble Approach or All Hot Air?

Keywords: geochronology, age dating, kimberlite, phlogopite, peridotite, lithosphere, argon

FLA_0062 
Authors

Abstract Title

File Name (.pdf)

AG Jones, JA Craven

Area Selection for Diamond Exploration using Deep-

Probing Electromagnetic Surveying

Keywords: Archean, slave, Western Superior, Canada, magnetotelluric

FLA_0278

AP Jones, AD Beard, HJ Milledge, G Cressey, C Kirk, P DeCarli New Nitride Mineral in Carbonado Diamond

Keywords: nitride, carbonado, Ti3CuN, CuTiN2, Cu3N, diamond, osbornite, nitrogen

FLA 0167

TC Junqueira-Brod, JA Brod, JC Gaspar, ESR Barbosa Magma-Sediments Interactions in the Aguas Emendadas Kamafugitic Diatremes, Goiás Alkaline Province, Brazil Keywords: peperite, kamafugite, Goiás Alkaline Province, Brazil, pyroclastic rocks, diatreme

FLA_0334

TC Junqueira-Brod, JC Gaspar, JA Brod, ESR Barbosa Magma Mixing in Cretaceous Kamafugites, Goias Alkaline Province, Brazil

Keywords: Aguas Emendadas, kamafugite, melaleucitite, Goiás Alkaline Province, magma mingling, magma mixing, pyroclastic rocks, diatreme

FLA_0338

FV Kaminsky, SM Sablukov, LI Sablukova, DMDeR Channer Late Proterozoic Kimberlites of Guaniamo, Venezuela: Anomalous, Ilmenite-Free, Mica Kimberlites of 'Isotopic Transitional' Type

Keywords: subvolcanic sheets, Nd-Sr, Lomonosov FLA 0001

FV Kaminsky, SM Sablukov, LI Sablukova

Diamondiferous Archaean Lamprophyres with Komatiitic Affinities from the Wawa Area, Ontario, Canada

Keywords: dyke, dike, Sandor, breccia

FLA 0391

E Kappus, EY Anthony, C Andronicos

Characterization of the Continental Mantle in an Active Rift Zone: Kilbourne Hole, New Mexico

Keywords: fertile lherzolite, protogranular, fine-grained equigranular tabular, porphyroclastic, geothermometry FLA 0192

RL Kellett, R Zahynacz, G Steensma

The Role of Borehole Geophysics in Improving the

Geophysical Imaging of Kimberlites in a Sedimentary Setting: Alberta, Canada

Keywords: Mountain Lake Intrusion, seismic, electromagnetics, gravity, magnetic, resistivity FLA_0015
Authors

Abstract Title

File Name (.pdf)

GK Khachatryan, FV Kaminsky

A Correlation between the Distribution of Nitrogen Centers in Diamonds and their Internal Structure and Mechanism of Growth

Keywords: FTIR, A-centre, B-centre, sectorial , A-center,

B-center

FLA_0002

BA Kjarsgaard

Behaviour of Kimberlite Magma in the Upper Crust and at Surface

Keywords: volatiles, paleo environment, carbon dioxide, water FLA_0294

IM Kjarsgaard, MB McClenaghan, BA Kjarsgaard, L Heaman Mineralogy of Kimberlite Boulders from Eskers in the Kirkland Lake and Lake Timiskaming Areas, North-eastern Ontario, Canada

Keywords: Mg-ilmenite, garnet, U/Pb age, glacial transport FLA_0292

O Klein-BenDavid, ES Izraeli, O Navon

Volatile-Rich Brine and Melt in Canadian Diamonds

Keywords: fibrous, micro-inclusions, Diavik, carbonatitic FLA_0109

O Klein-BenDavid, AM Logvinova, ES Izraeli, NV Sobolev, O Navon

Sulfide Melt Inclusions in Yubileinaya (Yakutia) Diamonds Keywords: Carbonatite, Siberia, micro-inclusions, fibrous, octahedral, sulphide

FLA_0111

S Klemme

The Garnet-Spinel Transition in Refractory Mantle Compositions

Keywords: garnet, spinel, knorringite, experimental petrology FLA 0055

M Kolebaba

Deep Infill Crater Model for Lac de Grass Kimberlites: Implications for Diamond Distribution

Keywords: crater, kimberlite emplacement, epiclastic

kimberlite, mass flow, volcaniclastic kimberlite

FLA_0340

MR Kolebaba, GH Read, BH Kahlert, D Kelsch

Diamondiferous Kimberlites on Victoria Island, Canada: A

Northern Extension of the Slave Craton

Keywords: Victoria Island, websterite, eclogite, peridotite, geotherm, Slave Craton, thermobarometry, Galaxy Kimberlite trend

FLA_0308 
Authors

Abstract Title

File Name (.pdf)

MG Kopylova

Two Distinct Origins of the Northern Slave Eclogites

Keywords: Slave craton, eclogite, REE, eclogite fabric, low-P mafic cumulate, mafic intrusive, metasomatism

FLA_0194

MG Kopylova, C McCammon

Composition and the Redox State of the Slave Peridotitic Mantle

Keywords: Slave craton, thermal state, mantle terrane, bulk composition, peridotite, oxygen fugacity, chemical stratification, mantle xenolith

FLA_0195

SI Kostrovitsky, EM Verichev, VK Garanin, LV Suvorova, IV Aschepkov, V Malkovets, WL Griffin

Megacrysts from Griba Kimberlite Pipe (Arkhangelsk

Province)

Keywords: high-Cr paragenesis, ilmenite, garnet, clinopyroxene

FLA 0074

SI Kostrovitsky, NV Alymova, AS Ivanov, VP Serov

Structure of the Daldyn Field (Yakutian Province) Based on the Study of Picroilmenite Composition

Keywords: taxon, pipe, cluster of pipes, magma chamber

FLA_0207

Y Kozai, M Arima

Diamond Dissolution in Kimberlite and Lamproite Melts at Deep Crustal Conditions

Keywords: diamond, dissolution, kimberlite, lamproite, morphology

FLA_0358

IN Kryvoshlyk

Garnet and Ilmenite Geochemical Computer Programs for Exploration for Diamonds

Keywords: garnet, ilmenite, chemical composition, diamond, exploration

FLA_0023

SM Kuehner, AJ Irving, HE O'Brien

A Kalborsite-Pitiglianoite-Kalsilite-Shcherbakovite-

Barytolamprophyllite-Wadeite-Bearing Lamproitic Dike

from the Northern Highwood Mountains, Montana, USA

Keywords: pseudoleucite, orangeite, perpotassic, potassium richterite, sodalite, tetraferriphlogopite, dyke

FLA_0047
Authors

Abstract Title

File Name (.pdf)

SS Kuligin, VG Malkovets, NP Pokhilenko, MA Vavilov, WL Griffin, SY O'Reilly

Mineralogical and Geochemical Characteristics of a Unique

Mantle Xenolith from the Udachnaya Kimberlite Pipe

Keywords: mineral, geochemistry, garnet, REE, rare earth elements

FLA_0114

S Kurszlaukis, W Barnett

Volcanological and Structural Aspects of the Venetia

Kimberlite Cluster

Keywords: volcanology, TKB, pyroclastic, emplacement, phreatomagmatism, structure, control, pipe

FLA_0063

Y Lahaye, GP Brey

Scale and Timing Constraints of Chemical Redistribution between Minerals of a Composite Garnet

Peridotite/Orthopyroxenite

Keywords: xenolith, trace elements, exsolution, laser ablation FLA_0186

AP le Roex, DR Bell, P Davis

Petrogenesis of Kimberley Group I Hypabyssal Kimberlites: Evidence from Bulk Rock Geochemistry

Keywords: lithosphere, magma, South Africa, plume

FLA_0097

J Ledo, AG Jones, JA Craven

Electrical Parameter Maps of Canada

Keywords: Canada, resistivity, magnetotellurics, anisotropy,

lithosphere

FLA 0280

DC Lee, J Maddren, BJ Griffin

The Importance of Chromite Morphology in Diamond Exploration

Keywords: plastic deformation, weathering, surface features, chemistry, resorption, elongation, kimberlite, lamproite

FLA 0315

NS Lefebvre, MG Kopylova, KR Kivi, RL Barnett

Diamondiferous Volcaniclastic Debris Flows of Wawa, Ontario, Canada

Keywords: diamonds, calc-alkaline lamprophyre, upper greenschist facies metamorphism, Superior craton, Michipicoten greenstone belt, oscillatory zoning, Archean FLA_0298

ML Lehtonen, JS Marmo

Glacial Dispersion Study of Kimberlitic Material in Quaternary Till from the Lahtojoki Pipe, Eastern Finland Keywords: diamond exploration, indicator mineral, geochemistry, Karelian Craton

FLA_0024 
Authors

Abstract Title

File Name (.pdf)

ML Lehtonen, HE OBrien, P Peltonen, BS Johanson, LK Pakkanen

Layered Mantle at the Edge of the Karelian Craton: P-T of Mantle Xenocrysts and Xenoliths from Eastern Finland Kimberlites

Keywords: Fennoscandia, lithosphere, thermobarometry, diamond

FLA_0260

NG Lensky, RW Niebo, JR Holloway, V Lyakhovsky, O Navon Bubble Nucleation as a Trigger for Xenolith Entrapment in Mantle Melts

Keywords: magma, volatiles, dike, kimberlite, dyke FLA 0108

I Leost, T Stachel, GP Brey, JW Harris

An Unusual Suite of Inclusions in Diamonds from Namibia Keywords: pyroxenes, intergrowths, exsolution, carbonates, barometry, thermometry, metasomatism FLA_0176

JPJ Letendre, M L'Heureux, TE Nowicki, R Creaser The Wemindji Kimberlites: Exploration and Geology Keywords: age, Canada, Quebec, indicator minerals, sill FLA 0311

KD Litasov, YD Litasov, VG Malkovets, H. Taniguchi Lithosphere Structure and Thermal Regime of the Upper Mantle Beneath the Baikal Region: Evidence from DeepSeated Xenoliths

Keywords: mantle, xenolith, geotherm, peridotite, pyroxenite, lithosphere, Baikal

FLA_0030

KD Litasov, E Ohtani

Hydrous Lower Mantle: The Water Source for Wet Plumes Keywords: mantle, perovskite, ferropericlase, water FLA 0031

GD Lockhart, H Grutter, JA Carlson

Temporal and Geomagnetic Relationship of Ekati Economic Kimberlites

Keywords: Lac de Gras, Slave, Rb-Sr, U-Pb, model ages

FLA_0284

AM Logvinova, O Klein-BenDavid, ES Israeli, O Navon, NV Sobolev

Microinclusions in Fibrous Diamonds from Yubileinaya Kimberlite Pipe (Yakutia)

Keywords: high pressure, upper mantle, sulfides, spectroscopy, fluid, carbonatitic melt, sulphide

FLA 0025
Authors

Abstract Title

File Name (.pdf)

V Lorenz, S Kurszlaukis

Kimberlite Pipes: Growth Models and Resulting

Implications for Diamond Exploration

Keywords: maars, diatremes, root zones, emplacement, target, strategy, model, phreatomagmatism

FLA_0036

IL Mahotkin, H Downes, E Hegner, AD Beard

Devonian Dike Swarms of Alkaline, Carbonatitic and

Primitive Magma-Type Rocks from the South Kola

Peninsula: Geochemical and Isotopic Constraints on

Plume-Lithospheric Interaction

Keywords: melilitite, alnoite, picrite, kimberlite, aillikite,

Kandalaksha, dike, damkjernite, dyke

FLA_0346

IL Mahotkin, J Robey, S Kurszlaukis, EP Valuev, NF Pylaev

Pipe Emplacement Model of the Lomonosov Diamond

Deposit, Arkhangelsk Region, NW Russia

Keywords: kimberlite, diatreme, maar, crater, pyroclastic, tuffisitic, volcano, Karpinskiy

FLA_0242

D Mainkar, B Lehmann, SE Haggerty

Discovery of the Very Large Crater-Facies Kimberlite System of Tokapal, Bastar District, Chhattisgarh, India Keywords: MKF, TKF, Bastar craton, lapilli tuff, Feeder, Tuff (ash) Beds, volcanic breccia

FLA_0068

VG Malkovets, LA Taylor, W Griffin, S O'Reilly, N Pearson, NP Pokhilenko, EM Verichev, NN Golovin, KD Litasov Cratonic Conditions Beneath Arkhangelsk, Russia: Garnet Peridotites from the Grib Kimberlite

Keywords: garnets peridotite, cratonic mantle, Arkhangelsk, Re/Os dating

FLA 0220

VG Malkovets, LA Taylor, W Griffin, S O'Reilly, NP Pokhilenko, EM Verichev, NN Golovin, KD Litasov, JW Valley, M Spicuzza Eclogites from the Grib Kimberlite Pipe, Arkhangelsk, Russia Keywords: eclogites, Arkhangelsk, oxygen isotopes, kimberlite FLA_0222

EV Malygina, NP Pokilenko, NV Sobolev

Coarse Peridotite Xenoliths of Udachnaya Kimberlite Pipe, Yakutia: Garnetization of Peridotites of the Central Siberian Platform Lithospheric Mantle

Keywords: garnet, lherzolite, spinel, harzburgite, garnetisation FLA_0191 
Authors

Abstract Title

File Name (.pdf)

KM Masun, BJ Doyle, SA Ball, S Walker

The Geology and Mineralogy of the Anuri Kimberlite, Nunavut, Canada

Keywords: Slave, petrography, Napaktulik, emplacement FLA 0246

D McBean, M Kirkley, C Revering

Structural Controls on the Morphology of the Snap Lake Kimberlite Dyke

Keywords: emplacement, Slave, craton, Northwest Territories, Canada, tectonics, Archean, extensional, dike

FLA 0258

CA McCammon, MG Kopylova

Mantle Oxygen Fugacity and Diamond Formation

Keywords: redox, Mossbauer spectroscopy, carbon isotopes,

Slave craton, garnet peridotite, fluids

FLA_0262

MB McClenaghan, IM Kjarsgaard, BA Kjarsgaard, PA Sobie Application of Surficial Exploration Methods in the Lake Timiskaming Kimberlite Field, Canada

Keywords: till, glaciated terrain, indicator minerals, till geochemistry, drift prospecting

FLA 0301

RE McElroy, TE Nowicki, DR Dyck, JA Carlson, JK Todd, SP Roebuck, B Crawford, S Harrison

The Geology of the Panda Kimberlite, Ekati Diamond Mine, Canada

Keywords: Lac de Gras, E174Contwoyto, Northwest Territories FLA 0197

N McKenna, JJ Gurney, JM Davidson

A Study of Diamonds, Diamond Inclusion Minerals and Other Mantle Minerals from the Swartruggens Kimberlite Dyke Swarm, South Africa

Keywords: macrocrysts, megacrysts, eclogitc inclusions, majorite, Type Ib, Group II kimberlite, peridotitic inclusions, websteritic inclusions, dike

FLA_0054

B McMonnies, J L Ward

Beyond Single-Sensor Magnetic Surveying. The Past, Present and Future of Airborne Magnetic Acquisition for Kimberlite Exploration

Keywords: airborne, geophysics, gradient

FLA 0268

S Mdludlu, MB Mabuza, KM Tainton, RJ Sweeney, C Skinner A Clinopyroxene Thermobarometry Traverse Across Coromandel Area, Brasil

Keywords: mantle, craton, geotherm, thermometry, nickel, garnet, adiabat

FLA_0282
Authors

Abstract Title

File Name (.pdf)

AH Menzies, M Frazenburg, MC Baumgartner, JJ Gurney, RO Moore

Evaluation of Chromites Derived from Kimberlites and Implications for Diamond Exploration Programs

Keywords: metasomatism, alteration, zonation, secondary electron imaging, backscatter electron imaging, elemental maps, mantle

FLA_0374

AH Menzies, K Westerlund, JJ Gurney, J Carlson, A Fung, T Nowicki

Peridotitic Mantle Xenoliths from Kimberlites on the Ekati Diamond Mine Property, NWT, Canada

Keywords: Slave Craton, lithosphere, geothermobarometry, paleogeotherm, Panda, diamond stability field

FLA_0305

RH Mitchell

Experimental Studies at 6 - 12 GPa of the Ondermatje Hypabyssal Kimberlite

Keywords: experimental petrology, spinel, ilmenite, clinopyroxene

FLA_0020

RH Mitchell, J Letendre

Mineralogy and Petrology of Kimberlite from Wemindji, Quebec

Keywords: kimberlite, sills, flow differentiation, macrocrysts, spinel, ilmenite, mica

FLA_0164

TS Mogg, MG Kopylova, BH Scott Smith, MB Kirkley Petrology of the Snap Lake Kimberlite, NWT, Canada Keywords: dyke, dike, hypabyssal, breccia, diamond, sheet, phlogopite

FLA_0067

J Moorhead, M Beaumier, R Girard, L Heaman

Distribution, Structural Controls and Ages of Kimberlite Fields in the Superior Province of Quebec

Keywords: kimberlite field, spacing, superior craton, age dates, lineaments, tectonic zones, rift basins

FLA_0275

T Morikiyo, SI Kostrovitsky, MWK Weerakoon, T Miyazaki, NV Vladykin, H Kagami, K Shuto

SR and ND Isotopic Difference Between Kimberlites and Carbonatites from Siberia

Keywords: kimberlite-carbonatite relationship, depleted mantle, Siberian Platform, subcontinental mantle FLA_0051 
Authors

Abstract Title

File Name (.pdf)

J Mustafa, TE Nowicki, P Oshust, D Dyck, B Crawford,

S Harrison

The Geology of the Misery Kimberlite, Ekati Diamond Mine, Canada

Keywords: Slave, Northwest, Lac de Gras, Contwoyto

FLA_0370

P Naidoo, J Stiefenhofer, M Field, R Dobbe

Recent Advances in the Geology of Koffiefontein Mine, Free State Province, South Africa

Keywords: kimberlite, underground, spinel, emplacement

FLA_0313

O Navon, ES Izraeli, O Klein-BenDavid

Fluid Inclusions in Diamonds - The Carbonatitic Connection

Keywords: brine, hydrous-silicic, micro-inclusions, melt

FLA_0107

A Nikiforova, AV Bobrov, VZ Spetsius

Garnet-Clinopyroxene Assemblage of Mantle Rocks from the

Obnazhennaya Kimberlite Pipe (Yakutia)

Keywords: eclogites, mantle, thermobarometry, pyroxenites

FLA_0149

GM Nowell, DG Pearson, DJ Jacob, ZV Spetsius, PH Nixon,

SE Haggerty

The Origin of Alkremites and Related Rocks: $A \mathrm{LU}-\mathrm{HF}$,

RB-SR and SM-ND Isotope Study

Keywords: garnet, spinel, eclogite, corganite, corgaspinite

FLA_0271

TE Nowicki, B Crawford, D Dyck, J Carlson, R McElroy,

H Helmstaedt, P Oshust

A Review of the Geology of Kimberlite Pipes of the Ekati

Property, Northwest Territories, Canada

Keywords: Lac de Gras, Slave, volcanology, pipe emplacement

FLA_0201

HE O'Brien, ML Lehtonen, RG Spencer, AC Birnie

Lithospheric Mantle in Eastern Finland: A $250 \mathrm{Km} 3 \mathrm{D}$

Transect

Keywords: Fennoscandia, kimberlite, xenocryst, Karelian

craton, diamond exploration

FLA_0261

SY O'Reilly, WL Griffin

Taking the Pulse of the Earth: Lithosphere Events Tracked by in-situ Geochronology

Keywords: mantle Re-Os ages, mantle sulfide, Kaapvaal craton, lithosphere formation, mantle evolution, mantle geophysics, lithosphere assembly, sulphide

FLA_0042
Authors

Abstract Title

File Name (.pdf)

DG Pearson, GM Nowell, DP Dowall, BA Kjarsgaard,

MG Kopylova, JA Armstrong

The Relative Roles of Lithosphere and Convecting Mantle in

Kimberlites from the Slave Province NWT: Constraints from

RE-OS Isotopes and Olivine Population Studies

Keywords: lamproites, Re-Os, potassic rocks, Hf isotopes

FLA_0269

NJ Pearson, WL Griffin, SY O'Reilly, G Delpech

Magnesium Isotopic Composition of Olivine from the

Lithospheric Mantle

Keywords: laser ablation, metasomatism, peridotite, Slave

Craton, Kaapvaal Craton, Kerguelen Islands, South-eastern

Australia

FLA_0129

JA Pell, OJ Ijewliw

Kimberlites, Melnoites and Look-Alikes in British

Columbia, Canada

Keywords: ultramafic lamprophyre, alkaline lamprophyre,

alkali basalt, ultrapotassic, pre-orogenic, aillikite

FLA_0200

P Peltonen

Platinum-Group Element Geochemistry of Eastern Finland

Kimberlites and their Mantle Sample

Keywords: melt, xenolith, PGE, mantle, diamond, sulphides,

Finland, Fennoscandia, sulfide

FLA_0052

BS Petrie, P Sheahan

The Sheahan-MDRU Diamond \& Mantle Rock Literature

Service

Keywords: library, internet, historical, technical literature

FLA_0008

D Phillips, JW Harrris

The Effect of Differential Mineral Compressibility on

Diamond Inclusion Thermobarometry

Keywords: mantle, geothermobarometry, garnet, pyroxene, temperature, pressure, kimberlite

FLA_0061

L Pizzolato, DJ Schulze

Geochemistry of Peridotites and Eclogites from the Kelsey

Lake Kimberlite, Colorado-Wyoming, USA

Keywords: Kelsey Lake, kimberlite, eclogite, peridotite

FLA 0300

LN Pokhilenko, AA Tomilenko, SS Kuligin, VV Khlestov

The Upper Mantle Heterogeneity: Thermodynamic

Calculations and Methods of Mathematical Statistics

Keywords: chromatography, fluid, xenolith, oxygen fugacity

FLA_0007 
Authors

Abstract Title

File Name (.pdf)

NP Pokilenko, AM Agashev, JA McDonald, NV Sobolev, SI Mityukhin, E Takazawa, MA Vavilov, YT Yanygin Kimberlites of the Nakyn Field, Siberia, and the Snap Lake/King Lake Dyke System, Slave Craton, Canada: A New Variety of Kimberlite with a Proposed Ultradeep Origin Keywords: carbonatite, lithosphere, mantle, majorite, Russia, dike

FLA_0172

NP Pokhilenko, WL Griffin, N Shimizu, RC McLean, VG Malkovets, LN Pokhilenko, EV Malygina

Pyropes and Chromites of the Snap Lake/King Lake Kimberlite Dyke System in Relation to the Problem of the Southern Slave Craton Lithospheric Mantle Structure and Composition

Keywords: Lithosphere, LREE, subcalcic, depleted, asthenosphere, metasomatism, dike

FLA_0171

NP Pokhilenko, JA McDonald, NV Sobolev, VN Reutsky, AE Hall, AM Logvinova, LF Reimers

Crystalline Inclusions and C-Isotope Ratios in Diamonds from the Snap Lake/King Lake Kimberlite Dyke System: Evidence of an Ultradeep and Enriched Lithospheric Mantle Keywords: majorite, chromite, lithosphere, Slave, olivine, dike FLA_0175

NP Pokhilenko, JA McDonald, MA Vavilov, AM Agashev, DB Clarke, K-J Wright

Kimberlites and Carbonatites of the Snap Lake/King Lake Dyke System: Structural Setting, Petrochemistry and Petrology of a Unique Type of Association

Keywords: phlogopite, groundmass, carbonate, hypabyssal, group 1, group 2, olivine, geochemical, dike

FLA_0183

NP Pokhilenko, DA Zedgenizov, VP Afanasiev, GM Rylov, HJ Milledge, A Jones, AE Hall, LF Reimers

Morphology and Internal Structure of Diamonds from the Snap Lake/King Lake Kimberlite Dyke System, Slave Craton, Canada

Keywords: cathodoluminescence, cube, coated, X-ray topography, dike

FLA_0181

YH Poudjom Djomani, SY O'Reilly, WL Griffin, BJ Doyle Geophysical Analysis of the Lithosphere Beneath the Slave Craton

Keywords: Slave craton gravity, Slave craton elastic thickness, slave craton deep structure, Slave craton kimberlite location, Slave craton lithosphere

FLA_0088
Authors

Abstract Title

File Name (.pdf)

D Prelevic, SF Foley, R Romer, V Cvetkovic

Serbian Tertiary Ultrapotassic Province-Petrology,

Geochemistry and Geodynamic Significance

Keywords: lamproites, kamafugites, ultrapotassic,

Mediterranean, $\mathrm{Sr}-\mathrm{Nd}-\mathrm{Pb}$ isotopes

FLA_0165

RF Preston, RJ Sweeney

A Comparison of Clinopyroxene Thermobarometric

Techniques: Applied to Jwaneng, Orapa and Markt Kimberlites

Keywords: thermobarometry, clinopyroxene, Jwaneng, Orapa,

Markt

FLA_0053

W Pretorius, H Helmstaedt, K Kyser

Whole Rock Platinum Group Element Geochemistry of

Kimberlitic Rocks - a Window into the Nature of the

Diamondiferous Mantle

Keywords: diamonds, on craton, off craton, metasomatism FLA_0319

P Promprated, LA Taylor, C Floss, VG Malkovets, M Anand, WL Griffin, NP Pokhilenko, NV Sobolev

Diamond Inclusions from Snap Lake, NWT, Canada

Keywords: Slave Craton, in situ, garnet, clinopyroxene, REE, majorite

FLA_0082

RP Rapp, N Shimizu

On the Origin of Eclogite and Websterite Parageneses in the Cratonic Mantle, and their Relationship to TTG Granitoid Magmatism

Keywords: diamond inclusions, mantle xenoliths, sanukitoid, Archean cratons, continental crust, peridotite xenoliths FLA_0307

GH Read, HS Grutter, LDS Winter, NB Luckman, GFM Gaunt Stratigraphic Relations, Kimberlite Emplacement and Lithospheric Thermal Evolution, Quiricó Basin, Minas Gerais State, Brazil

Keywords: clinopyroxene, thermobarometry, kamafugite, Areado, Mata da Corda, Cretaceous FLA_0304

TH Reddicliffe, J Jakimowicz, AJ Hell, JA Robins The Geology, Mineralogy, and Near Surface Characteristics of the Ashmore and Seppelt Kimberlite Clusters, North Kimberley Province, Australia

Keywords: kimberlite, geochemistry, chromite, diamond, infill, dyke, dike, cluster, Striker Resources

FLA_0139 
Authors

Abstract Title

File Name (.pdf)

S Rege, RM Davies, WL Griffin, S Jackson, SY O'Reilly

Trace Element Analysis of Diamond by LAM ICPMS:

Preliminary Results

Keywords: diamond formation, mantle fluids, diamond paragenesis, diamond fingerprints

FLA_0087

SH Richardson, SB Shirey, JW Harris

Episodic Diamond Genesis and Kaapvaal Craton Evolution

Keywords: Archean, peridotitic, eclogitic, sulfide, inclusion,

$\mathrm{Re}-\mathrm{Os}, \mathrm{Sm}-\mathrm{Nd}, \mathrm{Rb}-\mathrm{Sr}$, sulphide

FLA 0209

M. Roden, A. Patino-Douce, E. Laz'ko, E. Jagoutz

Exsolution Textures in High Pressure Garnets, Mir

Kimberlite, Siberia

Keywords: kimberlite, xenolith, majorite, ilmenite, ultrahigh pressure

FLA_0184

HA Rollo, HE Jamieson

Processed Kimberlite - Water Interactions in Diamond Mine Waste, Ekati Diamond Mine ${ }^{\mathrm{TM}}$, N.W.T., Canada

Keywords: aqueous geochemistry, environmental geochemistry, kimberlite tailings, sulphate

FLA_0286

L Rombouts

Distribution of Diamonds and Kimberlites on the Reguibat Craton, Mauritania

Keywords: Reguibat, G10, Archean, Jurassic, Eburnean FLA_0034

RL Rudnick, WF McDonough, PB Tomascak, T Zack

Lithium Isotopic Composition of Eclogites - Implications

for Subduction Zone Processes

Keywords: xenoliths, Koidu, Trescolmen, stable isotope, Man

Shield

FLA_0037

SM Sablukov, LI Sablukova

3-D Mapping of Mantle Substrate in the Zimny Bereg Area, Russia

Keywords: Arkhangelsk, kimberlite, mantle substrate, metasomatite, diamonds

FLA 0059

LI Sablukova, SM Sablukov, EM Verichev, NN Golovin

Mantle Xenoliths of the Grib Pipe, Zimny Bereg Area, Russia

Keywords: Arkhangelsk, kimberlite, mantle xenoliths, metasomatite, banded nodule

FLA_0060
Authors

Abstract Title

File Name (.pdf)

MD Schmitz, SB Shirey, RC Carlson

High-Precision U-Pb Geochronology and Lu-Hf Isotopic

Systematics of Zircons in Southern African Cratonic

Mantle Eclogites and Implications for Subcontinental

Lithospheric Mantle Evolution and Metasomatism

Keywords: hafnium, Ventersdorp, rifting, Gondwana

FLA 0252

DJ Schulze, B Harte, JW Valley, DMDeR Channer

Extreme Geochemical Variation Accompanying Diamond

Growth, Guaniamo, Venezuela

Keywords: carbon isotopes, oxygen isotopes, coesite, garnet, subduction, cathodoluminescence, zoning, geochemistry

FLA_0187

DJ Schulze, JW Valley, KS Viljoen, MJ Spicuzza

Oxygen Isotope Composition of Mantle Eclogites

Keywords: geochemistry, garnet, alkremite, subduction, South

Africa, kimberlite, laser fluorination

FLA_0188

HM Seitz, GP Brey, T Stachel, JW Harris

Lithium Abundances in Inclusions in Diamonds from the

Upper and Lower Mantle

Keywords: Lithium, inclusions in diamonds, eclogites, peridotites, websterites, majorite

FLA_0182

E.A. Shamshina, Z.A. Altukhova, S.A. Babushkina

Facial Characteristics of Kimberlite Rocks from the

Northern and Southern Parts of the Yakutian Kimberlite

Province (in the Light of the Duken and Daldyn Kimberlite

Fields)

Keywords: kimberlite breccia, geochronological dating,

mineral associations, phenocrysts, groundmass, petrochemical characteristics, autolith

FLA_0384

VV Sharygin, AV Golovin, NP Pokhilenko

Djerfisherite from Unaltered Kimberlites of the UdachnayaEastern Pipe, Yakutia

Keywords: sulphide, olivine inclusions, silicate-carbonate melt, sulfide

FLA_0227

VS Shchukin, SM Sablukov, LI Sablukova, EA Belousova, WL Griffin

Late Vendian Aerial Alkaline Volcanism in the Winter Coast Kimberlite Area (Arkhangelsk Diamondiferous Province) Keywords: Russian Platform, Kola-Dvina paleo-rift system, lamprophyre, volcanic, kimberlite, tuff, lava

FLA_0026 
Authors

Abstract Title

File Name (.pdf)

PN Shelkov

New Occurrences of Diamonds Indicator Minerals and Alkaline-Ultramafic Rocks from the Eastern Border of the Hanka Massif, Primorye, the Far East, Russia

Keywords: Nezametninskaya Diamond Area, Eastern Chinese rift system, Kedrovka river basin, aillikite

FLA 0243

M Shirasaka, E Takahashi

A Genesis of Carbonatitic Melt Within Subducting Oceanic Crusts: High-Pressure Experiments in the System MORB$\mathrm{CaCO3}$

Keywords: phase relations, subduction, carbonatite, carbonatebearing eclogite

FLA_0043

SB Shirey, SH Richardson, JW Harris

Integrated Models of Diamond Formation and Craton Evolution

Keywords: Kaapvaal, craton, isotopic age, southern Africa, Archean, seismic tomography, inclusion, lithosphere FLA_0267

A Shiryaev, ES Izraeli, E Hauri, EM Galimov, O Navon Fluid-Inclusions in Brazillian Coated Diamonds Keywords: brine, carbonatitic, hydro-silicic, melt, fibrous FLA_0115

SK Simakov

Garnet-Clinopyroxene and Clinopyroxene Geobarometry of Deep Mantle Eclogites and Peridotites

Keywords: pressure, temperature, thermodynamics, xenoliths FLA_0017

GJ Simandl

Diamond Potential in British Columbia, Canada?

Keywords: eclogite, alkaline province, ES-Diamond Model, diamondiferous mantle root, model, subduction, unconventional diamond

FLA 0368

NSC Simon, RW Carlson, GR Davies, GM Nowell, DG Pearson OS-SR-ND-HF Isotope Evidence for the Ancient Depletion and Subsequent Multi-Stage Enrichment History of the Kaapvaal Cratonic Lithosphere

Keywords: metasomatism, depleted lithosphere, isotopic disequilibrium, trace element disequilibrium

FLA 0117

EMW Skinner, DB Apter, C Morelli, I Tomlinson, KN Smithson Kimberlites of the Man Craton

Keywords: Guinea, Liberia, Sierra Leone, petrology, geochronology, tectonics, geomorphology

FLA_0225
Authors

Abstract Title

File Name (.pdf)

EMW Skinner, JS Marsh

Kimberlite Eruption Processes

Keywords: volcanology, diatreme, transitional, hypabyssal, pipe-formation

FLA_0040

AP Smelov, VF Timofeev, AI Zaitsev

A Geodynamic Model for the Formation of the North Asian

Craton in the Early Precambrian

Keywords: c+D15raton, Precambrian, crystalline basement, orogenic belts, kimberlites

FLA_0094

CB Smith, KM Sims, L Chimuka, AD Beard, R Townend Kimberlite Metasomatism at Murowa and Sese Pipes, Zimbabwe

Keywords: fenite, root-zone, emplacement, alkaline, fluids FLA_0121

DB Snyder, MG Bostock, GD Lockhart

Mapping the Mantle Lithosphere for Diamond Potential

Keywords: teleseismic surveys, Slave craton, mantle layers, anisotropy

FLA_0233

NV Sobolev, AM Logvinova, DA Zedgenizov, ES Yefimova, LA Taylor, P Promprated, VI Koptil, NN Zinchuk

Mineral Inclusions in Diamonds from Komsomolskaya and Krasnopresnenskaya Pipes, Yakutia: Evidence for Deep Lithospheric Heterogeneities in Siberian Craton

Keywords: upper mantle, high pressure, pyrope, garnet, majorite

FLA_0141

NV Sobolev, AM Logvinova, DA Zedgenizov, ES Yefimova Mineral Inclusions in Microdiamonds and Macrodiamonds from Kimberlites of Yakutia: A Comparative Study Keywords: pyrope, garnet, upper mantle, high pressure, majorite

FLA 0143

NV Sobolev, AM Logvinova, ES Yefimova, DA Zedgenizov, D.M.DeR. Channer

Polymineralic Eclogitic Inclusions in Guaniamo Diamonds, Venezuela: Evidence for Variable Diamond Growth Conditions Keywords: upper mantle, high pressure, coesite, garnet, paragenesis

FLA_0144

ZV Spetsius, LA Taylor

Metasomatic Diamonds in Eclogite Xenoliths: Petrologic and Photographic Evidence

Keywords: microdiamond genesis, young diamonds, partial melting, eclogites, metasomatism, metasomatic diamonds FLA_0064 
Authors

Abstract Title

File Name (.pdf)

ZV Spetsius

Highly Aluminous Xenoliths from Kimberlites of Yakutia:

Mantle Petrology Remarks

Keywords: eclogite, diamond, xenolith, kimberlite, Yakutia FLA_0065

T Stachel, S Aulbach, GP Brey, JW Harris, I Leost, R Tappert, KS Viljoen

Diamond Formation and Mantle Metasomatism: A Trace Element Perspective

Keywords: inclusion, garnet, cpx, REE, eclogitic, harzburgitic, lherzolitic, subduction, metasomatism

FLA_0390

LD Stasiuk, AR Sweet, DR Issler, K Kivi, GD Lockhart, DD Dyck Pre- and Post-Kimberlite Emplacement Thermal History of Cretaceous and Tertiary Sediments, Lac de Gras, Northwest Territories, Canada

Keywords: vitrinite reflectance, rock eval, palynology, porosity, maceral, fluorescence, maximum temperature

FLA_0056

J Stiefenhofer, DJ Farrow

Crater Deposits of the Mwadui Kimberlite

Keywords: Tanzania, De Beers, volcaniclastic, pyroclastic, open pit FLA 0160

GM Stott

Diabase Dyke Swarms as Structural Controls for Kimberlite Pipes Under the James Bay and Hudson Bay Lowlands, Ontario

Keywords: Attawapiskat, Kyle, Victor, Winisk, dike FLA_0317

MP Stubley

Spatial Distribution of Kimberlite in the Slave Craton: A Geometrical Approach

Keywords: Fry analysis, Lac de Gras, diabase dykes, diabase dikes, kimberlite clusters, alignment, corridors

FLA 0075

MP Stubley

Interpretive Compilation of Bedrock Geology of the Slave Craton

Keywords: GIS, diabase dykes, diabase dikes, faults, lineaments, fractures, domain analysis, kimberlite FLA_0076
Authors

Abstract Title

File Name (.pdf)

AR Sweet, LD Stasiuk, WW Nassichuk, O Catunneau,

DJ McIntyre

Paleontology and Diamonds: Geological Environments associated with Kimberlite Emplacement, Lac de Gras, Northwest Territories, Canada

Keywords: palynology, dinoflagellates, late Cretaceous, Palaeogene, paleogeography, spores, pollen FLA_0086

S Tappe, SF Foley, GA Jenner, B Ryan, D Besserer,

BA Kjarsgaard

Ultramafic Lamprophyre Dyke Swarm, Torngat Mountains, Quebec and Labrador: Mineralogy and Geochemistry

Keywords: Canada, aillikite, carbonatite, kimzeyite, Labrador Sea, heteromorphism, dike

FLA_0241

R Tappert, T Stachel, JW Harris, GP Brey

Mineral Inclusions in Diamonds from the Panda Kimberlite, Slave Province, Canada

Keywords: lower mantle, mantle petrology, North America, Lac de Gras, Northwest Territories

FLA_0101

LA Taylor, M Anand, P Promprated

Diamonds and their Inclusions: Are the Criteria for Syngenesis Valid?

Keywords: syngenesis, diamond inclusion, harzburgitic garnet, metasomatism, dating of diamond

FLA_0085

LA Taylor, ZV Spetsius, R Wiesli, M Anand, P Promprated, JW Valley

The Origin of Mantle Peridotites: Crustal Signatures from Yakutian Kimberlites

Keywords: Yakutian diamonds, carbon isotopes, oxygen isotopes, mineral inclusions, P-type diamonds, E-type diamonds

FLA_0077

EL Tomlinson, AP Jones, HJ Milledge, AD Beard

Multiple Fluids in Diamond Coat and their Role in Diamond Growth

Keywords: inclusions, mixing, synthesis, experimental FLA_0151

LA Tompkins, WA Taylor, DR Cowan

Diamond Prospectivity of the Altjawarra Craton, Australia

Keywords: North Australian Craton, magnetics, South

Georgina Basin, Siberia, SKIPPY, shromite, rutile dates, zircon dates

FLA_0251 
Authors

Abstract Title

File Name (.pdf)

P Ulmer, RJ Sweeney

Generation and Differentiation of Group II Kimberlites:

Constraints from High Pressure Experiments $10 \mathrm{Gpa}$

Keywords: orangeites, MARID, multi-anvil apparatus, phlogopite, carbonatites

FLA_0378

E Van Achterbergh, WL Griffin, SY O'Reilly, CG Ryan,

NJ Pearson, K Kivi, BJ Doyle

Melt Inclusions from the Deep Slave Lithosphere:

Constraints on the Origin and Evolution of Mantle-Derived Carbonatite and Kimberlite

Keywords: xenoliths, immiscibility, fluids, carbonate

FLA 0102

E Van Achterbergh, CG Ryan, WL Griffin, SY O'Reilly

Natural Trace Element Distribution between Immiscible

Silicate and Carbonate Melts Imaged by Nuclear Microprobe

Keywords: fractionation, kimberlite, REE, calcite, rare earth elements

FLA_0103

B van Coller, P Hildebrand, D Verran, F Barnes, TE Nowicki, M. Baumgartner, L Ott, JJ Gurney

Southern African Case Studies of Variations in Indicator Mineral Characteristics with Distance from Kimberlite Source Keywords: exploration, grain size, sample techniques, abrasion, surface textures, dispersion

FLA_0203

SK Verma

Geophysical Mapping of Kimberlite Pipes in South India

Keywords: geophysical, exploration, kimberlite, frequency and transient EM response, south India, challenges, geophysical surveys

FLA 0116

SK Verma

Geology, Geophysics, and Geodynamics of Extensive KCR Volcanism during Proterozoic Time in India

Keywords: kimberlites, lamproites, Rodinia, kimberlite

Volcanism, Proterozoic KCR's in India, Superplume

FLA_0217

KS (Fanus) Viljoen, Rene Dobbe

A Diamondiferous Lherzolite from the Premier Diamond Mine, South Africa

Keywords: mineral chemistry, peridotite, xenolith, infrared, nitrogen

FLA_0112

NV Vladykin, MI Lelyukh, AV Tolstov, VP Serov

Petrology of Kimberlite-Lamproite-Carbonatite Rock Association, East Prianabar'e (Russia)

FLA_0357
Authors

Abstract Title

File Name (.pdf)

K-L Wang, SY O'Reilly, WL Griffin, S-L Chung, W-S Juang Geochemical Characteristics of Mantle Xenoliths from Penghu Islands, Taiwan Straits, SE Asian Margin Keywords: subcontinental lithospheric mantle, southeastern China, metasomatism, mantle geochemistry, Proterozoic FLA_0071

KJ Webb, BH Scott Smith, JL Paul, CM Hetman Geology of the Victor Kimberlite, Attawapiskat, Northern Ontario, Canada: Cross-Cutting and Nested Craters Keywords: pyroclastic, juvenile lapilli, petrography, olivine phenocryst, hypabyssal, emplacement, bulk sampling, emplacement

FLA_0353

KJ Webb, J Stiefenhofer, M Field Overview of the Geology and Emplacement of the Jwaneng DK2 Kimberlite, Southern Botswana

Keywords: resedimented, volcaniclastic, pyroclastic, accretionary lapilli, emplacement, pipes FLA_0359

KJ Westerlund, EH Hauri, JJ Gurney FTIR Absorption and Stable Nitrogen and Carbon Isotope Microanalysis of Mid-Archaean Diamonds from the Panda Kimberlite

Keywords: cathodoluminescence, fractionation, sulfide, Inclusion, sulphide

FLA_0137

KJ Westerlund, SB Shirley, SH Richardson, JJ Gurney, JW Harris

RE-OS Isotope Systematics of Peridotitic Diamond Inclusion Sulfides from the Panda Kimberlite, Slave Craton Keywords: Archean, lithosphere, isochron, melt-depleted, sulphide

FLA_0134

K Whitehead, SH Richardson

A Geochemical Study of Peridotitic Diamonds from Premier Mine

Keywords: carbon, nitrogen, isotopes, aggregation, geothermobarometry

FLA_0154

C Williams, B Van Coller, T Nowicki, J Gurney

Mega Kalahari Geology: Challenges of Kimberlite

Exploration in this Medium

Keywords: desert, craton, super-swell, kimberlite, phanerozoic, sedimentary, South Africa, exploration

Keywords: desert, craton, super-swell, kimberlite, phanerozoic, sedimentary, South Africa, exploration

FLA_0006 
Authors

Abstract Title

File Name (.pdf)
Authors

Abstract Title

File Name (.pdf)

L Wilson, JW Head

Diatremes and Kimberlites 2: An Integrated Model of the Ascent and Eruptiion of Kimberlitic Magmas and the Production of Crater, Diatreme, and Hypabyssal Facies Keywords: dikes, sill, root zone, carbon dioxide, xenoliths, peridotite, dykes

FLA_0244

BA Wyatt, M Mitchell, SR Shee, WL Griffin, N Tomlinson, B White

The Brockman Creek Kimberlite, East Pilbara, Australia Keywords: mineral chemistry, trace elements, geotherm, diamond, spinel, garnet, apatite fission track, diamond indicator minerals

FLA_0180

AP Yelisseev, NP Pokhilenko, DA Zedgenizov, J Steeds Features of Coated Diamonds from the Snap Lake/King Lake Kimberlite Dyke System, Slave Craton, Canada, as Revealed by Optical Topography

Keywords: transmission, photoluminescence, growth, Raman, absorption spectroscopy, fibrous, nitrogen, dike

FLA_0179

JP Zonneveld, BA Kjarsgaard, SE Harvey, KY Marcia, D McNeil, LM Heaman, DJ White

Sedimentologic and Stratigraphic Constraints on Emplacement of the Star Kimberlite, East-Central Saskatchewan

Keywords: pyroclastic, volcaniclastic, primary kimberlite, secondary kimberlite, Cretaceous, Fort a la Corne, Canada, tephra

FLA_0296 